\title{
Anti-inflammatory and antioxidant activity of the traditional herbal formula Gwakhyangjeonggi-san via enhancement of heme oxygenase-1 expression in RAW264.7 macrophages
}

\author{
SOO-JIN JEONG ${ }^{1,2}$, OHN-SOON KIM ${ }^{1}$, SAE-ROM YOO ${ }^{3}$, CHANG-SEOB SEO $^{3}$, \\ YEJI KIM $^{3,4}$ and HYEUN-KYOO SHIN ${ }^{3}$ \\ ${ }^{1}$ KM Convergence Research Division, Korea Institute of Oriental Medicine, Daejeon 34054;
Korean Medicine Life Science, University of Science and Technology, Yuseong-gu, Daejeon 34113;
${ }^{3}$ K-herb Research Center, Korea Institute of Oriental Medicine, Daejeon, Chungcheong 34054;
${ }^{4}$ Mucosal Immunology Laboratory, Department of Convergence Medicine,
University of Ulsan College of Medicine/Asan Medical Center, Seoul 05505, Republic of Korea
}

Received April 8, 2015; Accepted December 21, 2015

DOI: $10.3892 / \mathrm{mmr} .2016 .5084$

\begin{abstract}
Gwakhyangjeonggi-san (GHJGS) is a mixture of herbal plants, including Agastache rugosa, Perilla frutescens, Angelica dahurica, Areca catechu, Poria cocos, Magnolia officinalis, Atractylodes macrocephala, Citrus reticulata, Pinellia ternata, Platycodon grandiflorum, Glycyrrhiza uralensis, Ziziphus jujuba and Zingiber officinale. GHJGS has been used for treating diarrhea-predominant irritable bowel syndrome in traditional Korean medicine. In the present study, the anti-inflammatory and antioxidant effects of GHJGS were investigated using the RAW 264.7 murine macrophage cell line. GHJGS significantly reduced production of the proinflammatory cytokines, tumor necrosis factor- $\alpha$, interleukin- 6 and prostaglandin $E_{2}$ in lipopolysaccharide (LPS)-stimulated macrophages. GHJGS markedly suppressed LPS-induced phosphorylation of mitogen-activated protein kinases, whereas it had no effect on nuclear factor- $\kappa \mathrm{B}$ activation. Furthermore, GHJGS enhanced expression of heme oxygenase- 1 and prevented the generation of reactive oxygen species in RAW 264.7 cells. These results indicate that GHJGS is a viable therapeutic agent against inflammation and oxidative stress-associated disorders.
\end{abstract}

Correspondence to: Dr Hyeun-Kyoo Shin, K-herb Research Center, Korea Institute of Oriental Medicine, 1672 Yuseong-daero, Yusung-gu, Daejeon, Chungcheong 34054, Republic of Korea E-mail: hkshin@kiom.re.kr

Key words: Gwakhyangjeonggi-san, anti-inflammation, antioxidant, heme oxygenase-1, macrophages

\section{Introduction}

Gwakhyangjeonggi-san (GHJGS) is a traditional Korean herbal formula composed of the following 13 medicinal herbs, Agastache rugosa, Perilla frutescens, Angelica dahurica, Areca catechu, Poria cocos, Magnolia officinalis, Atractylodes macrocephala, Citrus reticulata, Pinellia ternata, Platycodon grandiflorum, Glycyrrhiza uralensis, Ziziphus jujuba and Zingiber officinale. It has been used for treating diarrhea-predominant irritable bowel syndrome (1). In addition, GHJGS has been identified as an effective treatment for allergies (2), respiratory (3) and cardiovascular (4) diseases, and bacterial infections (5). However, to the best of our knowledge, there have been no reports to date on the anti-inflammatory effect of GHJGS.

Inflammation is a protective response against various harmful stimuli, such as pathogens, damaged cells and irritants (6). This response is controlled by production of proinflammatory biomolecules $(7,8)$. Overproduction of the proinflammatory cytokines, tumor necrosis factor- $\alpha$ (TNF- $\alpha$ ) and interleukin-6 (IL-6), and the proinflammatory mediator, prostaglandin $\mathrm{E}_{2}\left(\mathrm{PGE}_{2}\right)$ may result in inflammatory disorders accompanied by fever, tissue destruction or pain $(7,8)$. Therefore, targeting these proinflammatory cytokines or $\mathrm{PGE}_{2}$ is considered to be a potential therapeutic approach for treating inflammatory disorders. Mitogen-activated protein kinase (MAPK) and/or nuclear factor $-\kappa \mathrm{B}(\mathrm{NF}-\kappa \mathrm{B})$ signaling pathways are important in the regulation of inflammatory responses, including triggering the initiation of proinflammatory cytokine production (9). Additionally, previous studies have reported a link between anti-inflammatory and antioxidative regulation using various natural products through activation of heme oxygenase-1 (HO-1), an enzyme with antioxidant effects (10-12).

In the present study, the anti-inflammatory and antioxidant activity of GHJGS was investigated using the murine macrophage cell line, RAW 264.7. The inflammatory reaction was induced by lipopolysaccharide (LPS) stimulation and the 
production of TNF- $\alpha$, IL- 6 , and $\mathrm{PGE}_{2}$ was examined using enzyme-linked immunosorbent assays (ELISAs). In addition, the effects of GHJGS on activation of MAPK and NF- $\mathrm{KB}$ signaling pathways, and the expression of $\mathrm{HO}-1$ in RAW 264.7 cells were investigated.

\section{Materials and methods}

Plant materials. The 13 herbs that form GHJGS were purchased from Kwangmyungdang Medicinal Herbs (Ulsan, South Korea). The taxonomic classification of the 13 herbs was verified by Professor Je-Hyun Lee from Dongguk University (Gyeongju, South Korea). Voucher specimens (2012-KE32-1 to KE32-13) were deposited at the K-herb Research Center, Korea Institute of Oriental Medicine (Daejeon, South Korea).

Chemicals and reagents. Liquiritin, glycyrrhizin and 6-gingerol were purchased from Wako Pure Chemical Industries, Ltd. (Osaka, Japan). Hesperidin and rosmarinic acid were purchased from Acros Organics (Morris, NJ, USA) and Sigma-Aldrich (St. Louis, MO, USA), respectively. The purity of each component was determined to be $\geq 98 \%$ using high-performance liquid chromatography (HPLC) analysis. The chemical structures of the five marker compounds are presented in Fig. 1A. HPLC-grade reagents, methanol, acetonitrile and distilled water were obtained from J.T. Baker; Avanto Performance Materials (Phillipsburg, NJ, USA). Acetic acid was obtained from Merck KGaA (Darmstadt, Germany).

Preparation of GHJGS decoction. GHJGS was composed of 13 herbs (Table I; total weight, $5.0 \mathrm{~kg}, \sim 148.15$ times the composition of a single dose) and extracted in distilled water at $100^{\circ} \mathrm{C}$ for $2 \mathrm{~h}$ under $98 \mathrm{kPa}$ pressure using a COSMOS-660 electric extractor (KyungSeo Machine Co., Incheon, South Korea). The extracted solution was filtered using a standard sieve (no. 270; mesh size, $53 \mu \mathrm{m}$; Chung Gye Sang Gong Sa, Seoul, Korea) and freeze-dried. The yield of the extract was $12.89 \%$ (644.5 g). The lyophilized GHJGS extract (40 mg) was dissolved in $50 \%$ methanol $(20 \mathrm{ml})$ and mixed for quantitative analysis. The solution was filtered through a $0.2-\mu \mathrm{m}$ SmartPor GHP syringe filter (Woong Ki Science Co., Ltd., Seoul, South Korea) prior to being injected into a HPLC column.

Quantitative analysis of GHJGS. The quantitative determination was performed using a Prominence LC-20A series HPLC system (Shimadzu Corporation, Kyoto, Japan) consisting of a solvent delivery unit (LC-20AT), online degasser (DGU-20A3), column oven (CTO-20A), auto sample injector (SIL-20AC), and photodiode array (PDA) detector (SPD-M20A). Data were collected and processed using LC solution software (version 1.24; Shimadzu Corporation). A Gemini C18 column (250 mm x $4.6 \mathrm{~mm}$; particle size, $5 \mu \mathrm{m}$; Phenomenex, Inc., Torrance, CA, USA) was used for separation of the marker compounds and maintained at $40^{\circ} \mathrm{C}$. The mobile phases consisted of $1.0 \%(\mathrm{v} / \mathrm{v})$ acetic acid in distilled water (designated as A) and 1.0\% (v/v) acetic acid in acetonitrile (designated as $\mathrm{B}$ ). The gradient flow was as follows: 5-70\% B for 0-40 min; 70-100\% B for 40-50 min; 100\% B for $50-55 \mathrm{~min}$; and $100-5 \% \mathrm{~B}$ for $55-60 \mathrm{~min}$. The analysis was conducted at $1.0 \mathrm{ml} / \mathrm{min}$ with PDA detection at $254 \mathrm{~nm}$ (glycyrrhizin), $280 \mathrm{~nm}$ (liquiritin, hesperidin and 6-gingerol), and $330 \mathrm{~nm}$ (rosmarinic acid). The sample injection volume was $10 \mu \mathrm{l}$.

Cell culture. The murine macrophage cell line, RAW 264.7, was obtained from the American Type Culture Collection (Rockville, MD, USA). The cells were cultured in Dulbecco's modified Eagle's medium (Gibco; Thermo Fisher Scientific, Inc., Waltham, MA, USA) supplemented with 5.5\% heat-inactivated fetal bovine serum (Gibco; Thermo Fisher Scientific, Inc.), penicillin (100 U/ml; HyClone Laboratories, Inc., Logan, UT, USA), and streptomycin (100 $\mu \mathrm{g} / \mathrm{ml}$; HyClone Laboratories, Inc.) in an incubator with $5 \% \mathrm{CO}_{2}$ at $37^{\circ} \mathrm{C}$.

Cytotoxicity assay. Cell viability assay was performed to determine the cytotoxicity of GHJGS using a Cell Counting Kit-8 (CCK-8; Dojindo Molecular Technologies, Inc., Kumamoto, Japan). Cells were plated onto a 96-well microplate at $3 \times 10^{3}$ cells/well and treated with $0,15.625,31.25,62.5,125,250,500$ or $1,000 \mu \mathrm{g} / \mathrm{ml}$ GHJGS for $24 \mathrm{~h}$. Following incubation with CCK- 8 reagent for $4 \mathrm{~h}$, optical density (OD) at a wavelength of $450 \mathrm{~nm}$ was determined using a Benchmark plus microplate reader (Bio-Rad Laboratories, Inc., Hercules, CA, USA). Cell viability was calculated using the following equation: Cell viability $(\%)=$ mean $\mathrm{OD}_{\text {GHGJS-treated cells }} /$ mean $\mathrm{OD}_{\text {untreated }}$ cells $\mathrm{x} 100$

ELISAs for TNF- $\alpha, I L-6$, and $P G E_{2}$. Cells were pretreated with $0,250,500$ or $1,000 \mu \mathrm{g} / \mathrm{ml}$ GHJGS for $4 \mathrm{~h}$ and stimulated with LPS $(1 \mu \mathrm{g} / \mathrm{ml})$ for an additional $20 \mathrm{~h}$. Production of TNF- $\alpha$, IL-6 and PGE 2 in the culture supernatants was measured using commercial ELISA kits from R\&D Systems, Inc. (Minneapolis, MN, USA), BD Biosciences (San Jose, CA, USA) and Cayman Chemical Company (Ann Arbor, MI, USA), respectively. Indomethacin ( $2.5 \mathrm{ng} / \mathrm{ml}$; Sigma-Aldrich) was used as a positive control.

Western blotting. Whole cell extract (WCE) was prepared by suspending cells using the Mammalian Cell Lysis kit (Sigma-Aldrich) containing protease inhibitor cocktail (Roche Diagnostics, Indianapolis, IN, USA). Nuclear extract (NE) was isolated using NE-PER ${ }^{\circledR}$ Nuclear and Cytoplasmic Extraction Reagents (Thermo Fisher Scientific, Inc., Rockford, IL, USA) according to the manufacturer's protocol. The protein concentration was determined using the Bio-Rad Protein Assay kit II (Bio-Rad Laboratories, Inc.). Equal quantities of cell extract $(30 \mu \mathrm{g})$ were resolved by $4-20 \%$ sodium dodecyl sulfate-polyacrylamide gel electrophoresis (Bio-Rad Laboratories, Inc.) at $100 \mathrm{v}$ for $1 \mathrm{~h}$ and transferred to a polyvinylidene fluoride membrane (GE Healthcare Life Sciences, Piscataway, NJ, USA). The membrane was incubated with blocking solution [5\% skimmed milk in Tris-buffered saline containing Tween-20 (TBST); DyneBio, Seongnam, Korea], followed by an overnight incubation at $4^{\circ} \mathrm{C}$ with the appropriate primary antibody, including rabbit polyclonal phosphorylated (p)-p38 MAPK (1:1,000 dilution; cat. no. 9211; Cell Signaling Technology, Inc., Danvers, MA, USA), rabbit polyclonal p-extracellular signal-regulated kinase (ERK; 1:1,000 dilution; cat. no. 9101; Cell Signaling Technology, Inc.), rabbit polyclonal p-c-Jun N-terminal kinase (JNK; 1:1,000 
Table I. Composition of Gwakhyangjeonggi-san.

\begin{tabular}{llll}
\hline Latin name & \multicolumn{1}{c}{ Scientific name } & Quantity (g) & Origin \\
\hline Agastachis Herba & Agastache rugosa (Fisch. et Meyer) O. Kuntze & 833.30 & Andong, South Korea \\
Perillae Herba & Perilla frutescens var. crispa (Thunb.) H. Deane & 555.56 & Yeongcheon, South Korea \\
Angelicae Dahuricae Radix & Angelica dahurica Benth. et Hook. f. & 277.78 & Uljin, South Korea \\
Arecae Pericarpium & Areca catechu L. & 277.78 & China \\
Hoelen & Poria cocos F. A. Wolf & 277.78 & Pyeongchang, South Korea \\
Magnoliae Cortex & Magnolia officinalis Rehd. et E. H. Wils. & 277.78 & China \\
Atractylodis Rhizoma Alba & Atractylodes macrocephala Koidz. & 277.78 & China \\
Citri Unshius Pericarpium & Citrus reticulata Blanco & 277.78 & Jeju, South Korea \\
Pinelliae Tuber & Pinellia ternata Breit. & 277.78 & China \\
Platycodi Radix & Platycodon grandiflorum A. DC. & 277.78 & Andong, South Korea \\
Glycyrrhizae Radix et Rhizoma & Glycyrrhiza uralensis Fisch. & 277.78 & China \\
Zizyphi Fructus & Ziziphus jujuba var. inermis (Bunge) Rehder & 555.56 & Yeongcheon, South Korea \\
Zingiberis Rhizoma Crudus & Zingiber officinale Rosc. & 555.56 & Ulsan, South Korea \\
Total & & 5,000 & \\
\hline
\end{tabular}

dilution; cat. no. 9251; Cell Signaling Technology, Inc.), mouse monoclonal HO-1 (1:1,000 dilution; cat. no. ab13248; Abcam, Boston, MA, USA), rabbit polyclonal NF-кB p65 (1:1,000; cat. no. sc-372; Santa Cruz Biotechnology, Inc., Dallas, TX, USA) and mouse monoclonal $\beta$-actin (1:5,000; cat. no. sc-47778; Santa Cruz Biotechnology, Inc.). The membranes were washed three times with TBST, and then incubated with polyclonal horseradish peroxidase-conjugated goat anti-mouse IgG (cat. no. 115-001-003; Jackson ImmunoResearch Laboratories, Inc., West Grove, PA, USA; 1:2,000 dilution) and goat anti-rabbit IgG (cat. no. 111-001-003; Jackson ImmunoResearch Laboratories, Inc.; 1:2,000 dilution) secondary antibodies for $1 \mathrm{~h}$ at room temperature. The membranes were washed three times with TBST, and developed using the SuperSignal West Pico Chemiluminescent Substrate (Thermo Fisher Scientific, Inc.). Image capture was performed using Chemi-Doc (Bio-Rad Laboratories, Inc.).

Reactive oxygen species (ROS) staining. To examine the generation of ROS, the ROS-ID ${ }^{\mathrm{TM}}$ Total ROS Detection kit (Enzo Life Sciences, Inc., Plymouth Meeting, PA, USA) was used. The effect of GHJGS on ROS generation was examined by immunofluorescence staining. Cells were plated on $\mu$-Dishes $35 \mathrm{~mm}$ (Ibidi, Aarhus, Denmark), treated with GHJGS $(1,000 \mu \mathrm{g} / \mathrm{ml})$ and LPS $(1 \mu \mathrm{g} / \mathrm{ml})$ for $30 \mathrm{~min}$, and fixed in $4 \%$ paraformaldehyde (Sigma-Aldrich) and $100 \%$ acetone (Sigma-Aldrich). The ROS detection solution was loaded to the cells and incubated at room temperature for $1 \mathrm{~h}$. Following the addition of a mounting medium (Vector Laboratories Inc., Burlingame, CA, USA), the stained cells were visualized using a FLUOVIEW FV10i confocal microscope (Olympus Corporation, Tokyo, Japan).

Statistical analysis. The data are expressed as the mean \pm standard error of the mean. Data were analyzed using one-way analysis of variance and Dunnett's multiple comparisons test. $\mathrm{P}<0.05$ was considered to indicate a statistically significant difference.

\section{Results}

Quantitative determination of the five marker compounds in GHJGS. The novel HPLC-PDA method was applied for simultaneous quantification of the five marker compounds in GHJGS. The typical chromatogram patterns for standard compounds and the GHJGS decoction are presented in Fig. 1B and $\mathrm{C}$. The retention times of the liquiritin, hesperidin, rosmarinic acid, glycyrrhizin and 6-gingerol were $~ 17.35,19.35$, $20.66,33.39$, and $33.88 \mathrm{~min}$, respectively. The concentrations of the five components were between 1.18 and $3.16 \mathrm{mg} / \mathrm{g}$ (Table II).

GHJGS inhibits production of TNF- $\alpha, I L-6$ and $P G E_{2}$ in LPS-stimulated RAW 264.7 cells. Cytotoxicity of GHJGS was evaluated using RAW 264.7 cells. Cells were treated with serial dilutions of GHJGS for $24 \mathrm{~h}$. Fig. 2 demonstrates that no cytotoxic effect was observed up to $1,000 \mu \mathrm{g} / \mathrm{ml}$ GHJGS treatment. For the subsequent assays, cell treatment with GHJGS was performed in the nontoxic concentration range $(250-1,000 \mu \mathrm{g} / \mathrm{ml})$.

GHJGS reduces the levels of TNF- $\alpha$. IL- 6 and $P G E_{2}$ in $L P S$-stimulated RAW 264.7 cells. To examine the anti-inflammatory effect of GHJGS, production of TNF- $\alpha$ and IL-6 was assessed in LPS-stimulated RAW 264.7 cells. LPS stimulation significantly increased levels of TNF- $\alpha$ and IL-6 in RAW 264.7 cells, compared with untreated controls. By contrast, GHJGS treatment significantly reduced LPS-induced production of TNF- $\alpha$ and IL-6 (P<0.01; Fig. 3A and B). The quantity of $\mathrm{PGE}_{2}$ was also determined and indomethacin served as a positive control. The level of $\mathrm{PGE}_{2}$ was significantly increased in cells treated with LPS alone. By contrast, GHJGS treatment significantly reduced $\mathrm{PGE}_{2}$ production by LPS stimulation $(\mathrm{P}<0.01$; Fig. 3C).

GHJGS suppresses phosphorylation of MAPK family proteins in LPS-stimulated RAW 264.7 cells. As demonstrated by 
Table II. Concentration of the five marker compounds in Gwakhyangjeonggi-san by high-performance liquid chromatography.

\begin{tabular}{lcccl}
\hline & \multicolumn{3}{c}{ Concentration } & \\
\cline { 2 - 4 } Compound & Mean (mg/g; $\mathrm{n}=3)$ & $\mathrm{SD}$ & $\mathrm{RSD}(\%)$ & Source \\
\hline Liquiritin & 1.18 & 0.01 & 1.08 & Glycyrrhiza uralensis \\
Hesperidin & 3.16 & 0.01 & 0.27 & $\begin{array}{l}\text { Camellia reticulata } \\
\text { Agastache rugosa } \\
\text { Rosmarinic acid }\end{array}$ \\
and Perilla frutescens \\
Glycyrrhizin & 1.45 & 0.01 & 0.74 & Glycyrrhiza uralensis \\
6-Gingerol & 3.03 & 0.02 & 0.58 & Zingiber officinale \\
\hline
\end{tabular}

SD, standard deviation; RSD, relative standard deviation.

A<smiles>Oc1ccc2c(c1)OC(c1ccc(OCCOc3ccc(O)o3)cc1)C2</smiles>

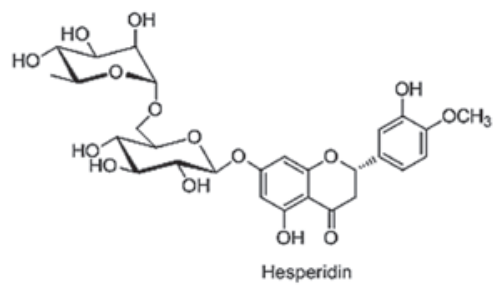<smiles>Oc1ccc(C=C2COC3OC(O)C(O)C3C2)c(O)c1</smiles>

Hesperidin
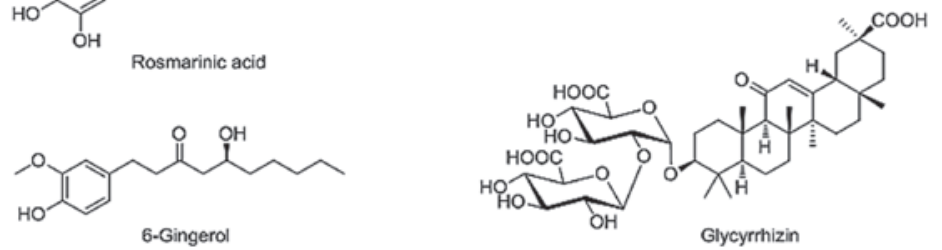

B

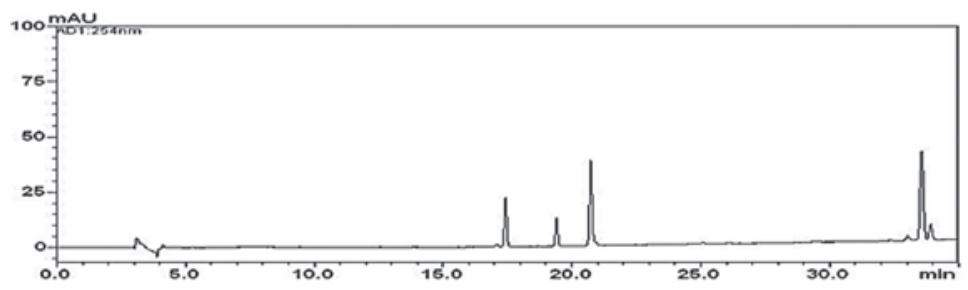

C

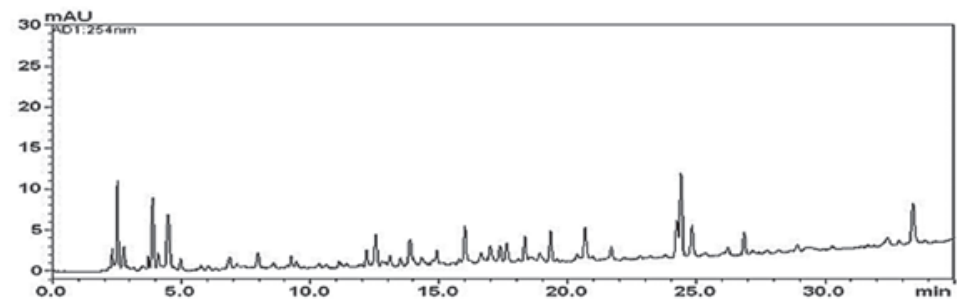

Figure 1. Chemical structures of (A) the five marker compounds, liquiritin, hesperidin, rosmarinic acid, 6-gingerol and glycyrrhizin. High-performance liquid chromatography chromatograms of the (B) standard mixture and (C) Gwakhyangjeonggi-san extract at $254 \mathrm{~nm}$.

Fig. 4A, LPS markedly enhanced phosphorylation of p38 MAPK, ERK and JNK in RAW 264.7 cells, compared with untreated controls. LPS-induced activation of MAPKs was inhibited when cells were pretreated with GHJGS. NF-кB p65 expression in the nucleus of RAW 264.7 cells was also analyzed. LPS increased the expression level of NF- $\mathrm{KB}$ $\mathrm{p} 65$, compared with that of untreated controls. When cells were exposed to LPS and GHJGS, the GHJGS exerted no influence on NF-кB p65 in the LPS-stimulated RAW 264.7 cells (Fig. 4B).

GHJGS induces HO-1 expression and blocks LPS-induced ROS generation in RAW 264.7 cells. Cells distinctly stained with green fluorescence were observed in LPS-stimulated cells compared with the undifferentiated control (Fig. 5A). By contrast, GHJGS treatment blocked LPS-mediated ROS 


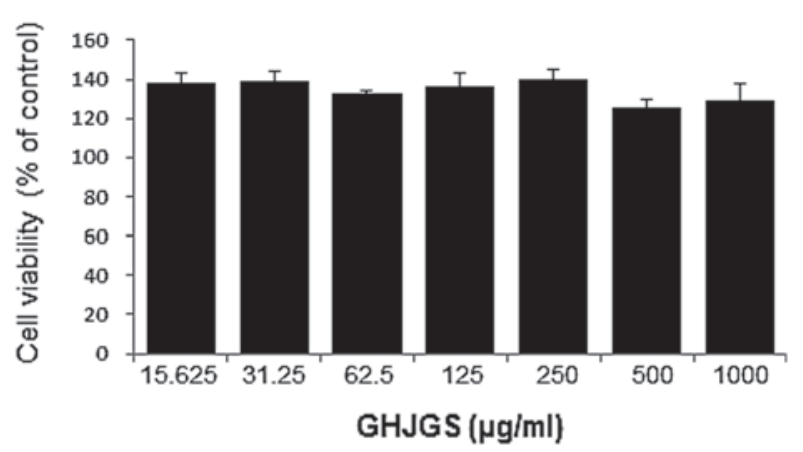

Figure 2. Cytotoxic effect of GHJGS on RAW 264.7 cells. Cells were plated onto 96-well microplates and treated with various concentrations of GHJGS for 24 h. Cell viability (\%) was assessed using a Cell Counting Kit-8 assay. The values are expressed as the mean \pm standard error of three independent experiments. GHJGS, Gwakhyangjeonggi-san.
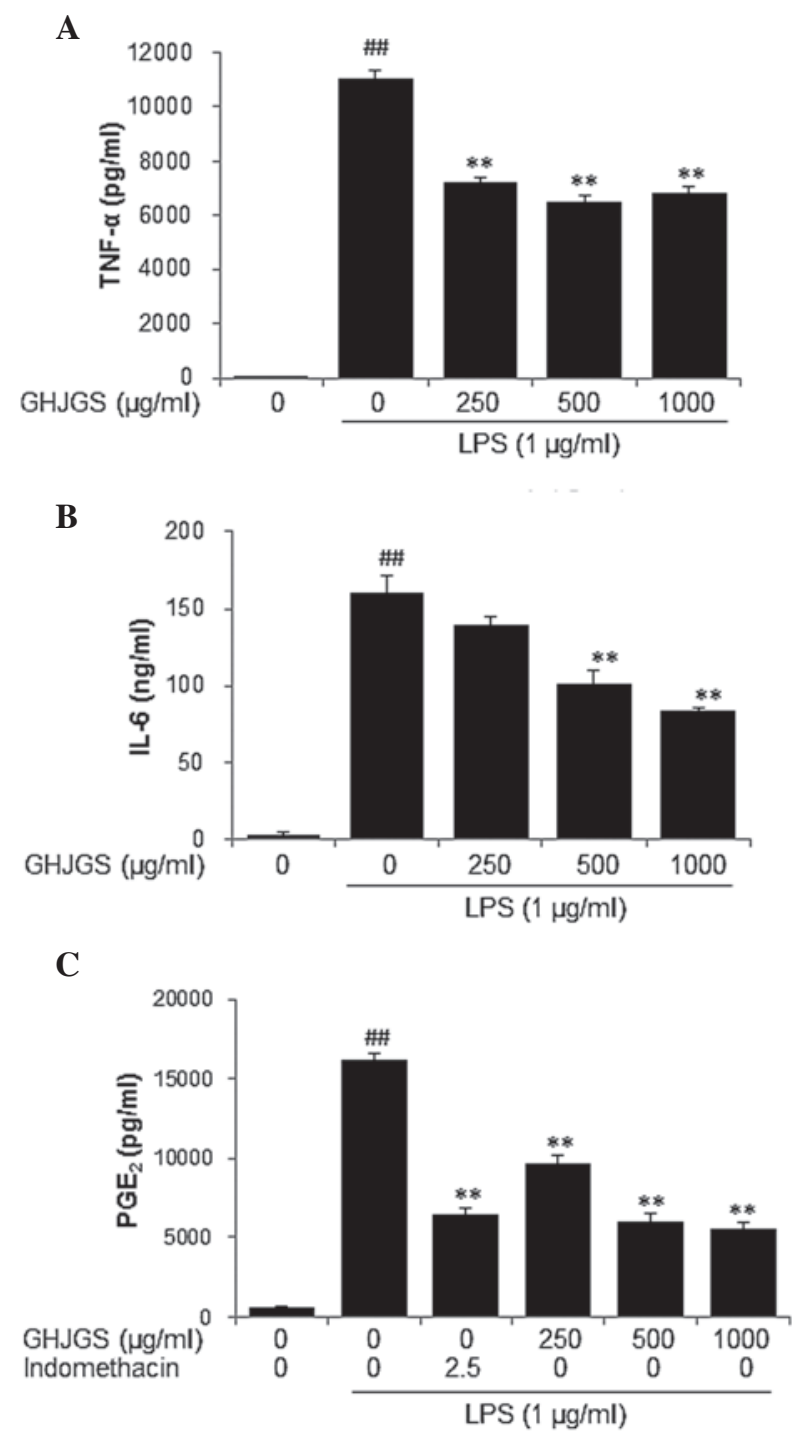

Figure 3. Effects of GHJGS on production of TNF- $\alpha$, IL- 6 and $\mathrm{PGE}_{2}$ in LPS-stimulated RAW 264.7 cells. Levels of (A) TNF- $\alpha$, (B) IL-6 and (C) $\mathrm{PGE}_{2}$ were estimated by enzyme-linked immunosorbent assays. Cells were pretreated with a range of different GHJGS concentrations for $4 \mathrm{~h}$ and exposed to LPS for an additional $20 \mathrm{~h}$. The bar graphs represent the mean \pm standard error of three independent experiments. ${ }^{\# \#} \mathrm{P}<0.01$ vs. vehicle control cells; ${ }^{* *} \mathrm{P}<0.01$ vs. LPS-treated cells. TNF- $\alpha$, tumor necrosis factor- $\alpha$; GHJGS, Gwakhyangjeonggi-san; LPS, lipopolysaccharide; IL-6, interleukin-6; $\mathrm{PGE}_{2}$, prostaglandin $\mathrm{E}_{2}$.

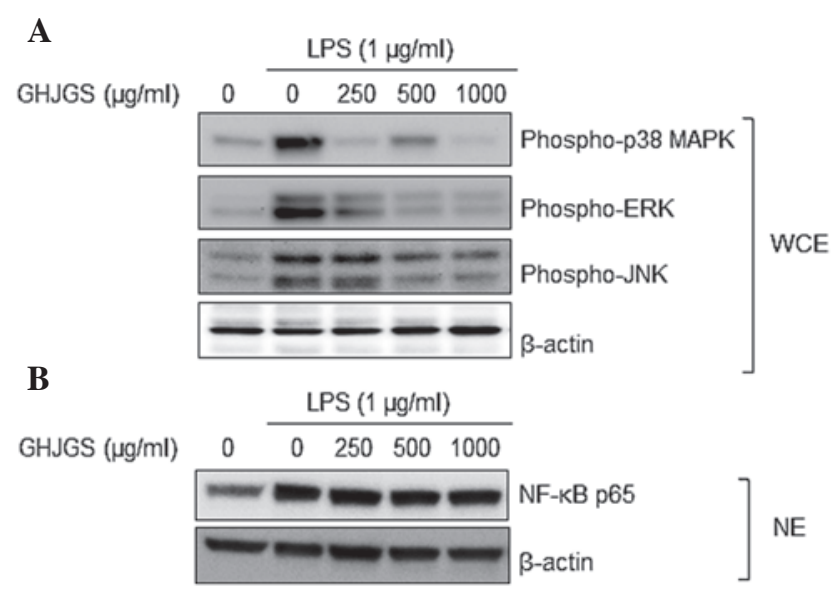

Figure 4. Effect of GHJGS on the activation of MAPKs and NF- $\kappa B$ in LPS-stimulated RAW 264.7 cells. (A) WCEs were prepared and subjected to western blotting to detect phosphorylation of p38 MAPK, ERK and JNK. (B) NEs were isolated and subjected to western blotting for NF- $\mathrm{\kappa B}$ p65 detection. GHJGS, Gwakhyangjeonggi-san; LPS, lipopolysaccharide; MAPK, mitogen-activated protein kinase; ERK, extracellular signal-regulated kinase;

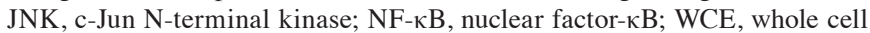
extract; NE, nuclear extract.
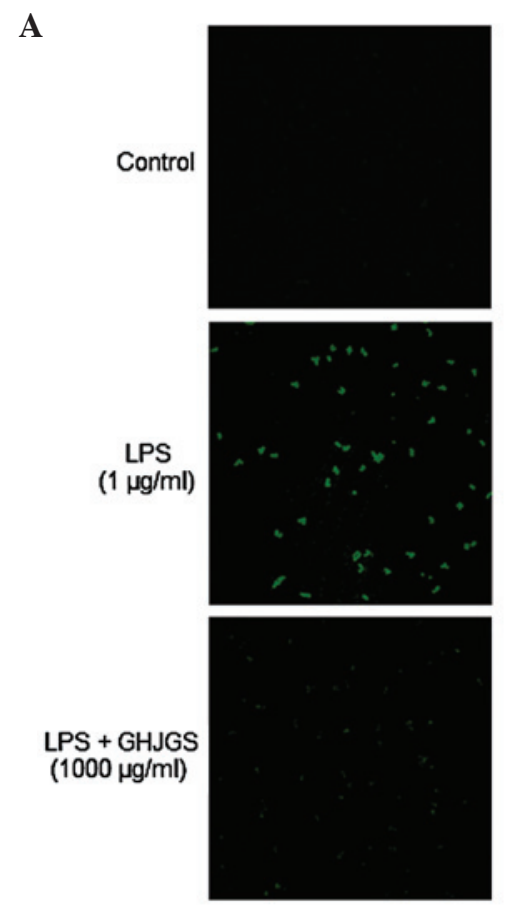

B

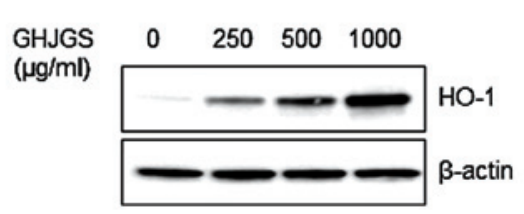

Figure 5. Effects of GHJGS on ROS generation and HO-1 expression in RAW 264.7 cells. (A) Cells were pretreated with GHJGS $(1,000 \mu \mathrm{g} / \mathrm{ml})$ for $4 \mathrm{~h}$ and stimulated with LPS $(1 \mu \mathrm{g} / \mathrm{ml})$ for $30 \mathrm{~min}$. Cells were fixed in $4 \%$ paraformaldehyde, stained with Oxidative Stress Detection Reagent and visualized under an Olympus FLUOVIEW FV10i confocal microscope (x100). GHJGS reduced LPS-mediated ROS generation stained with green fluorescence. (B) Cells were treated with 250,500 or 1,000 $\mu \mathrm{g} / \mathrm{ml}$ GHJGS for $24 \mathrm{~h}$. Whole cell extracts were prepared and subjected to western blotting for HO-1. LPS, lipopolysaccharide; GHJGS, Gwakhyangjeonggi-san; HO-1, heme oxygenase-1; ROS, reactive oxygen species. 
generation (Fig. 5A). Additionally, GHJGS induced HO-1 expression in a dose-dependent manner (Fig. 5B).

\section{Discussion}

Although the global market share for synthetic therapeutic agents is gradually increasing annually, various negative features, including toxicity and severe side effects, may limit their therapeutic efficacies and result in reduced quality of life $(13,14)$. To overcome the issues associated with synthetic therapeutic agents, natural products (including herbal medicines) have been considered as a valuable source for establishing novel remedies for numerous years $(15,16)$. Therefore, the present study aimed to determine the anti-inflammatory and antioxidant effects of the herbal formula, GHJGS using in vitro experimental models. GHJGS suppressed LPS-stimulated production of TNF- $\alpha$, IL-6 and $\mathrm{PGE}_{2}$, and inhibited LPS-mediated phosphorylation of MAPKs. GHJGS increased the scavenging activity of 2,2'-azino-bis(3-ethylbenzothiazoline-6-sulphonic acid) and di(phenyl)-(2,4,6-trinitrophenyl)iminoazanium radicals, and reduced low-density lipoprotein oxidation (data not shown). In addition, GHJGS enhanced the level of HO-1 expression and reduced the LPS-induced generation of ROS in RAW 264.7 macrophages.

Table I presents the relative quantities and origin of the 13 herbs that form GHJGS. To improve quality control of GHJGS, simultaneous analysis of the marker compounds in GHJGS were conducted using the HPLC-PDA method. The primary active ingredients of each herb are as follows: Phenylpropanoids (e.g. rosmarinic acid) from A. rugose (17), phenylpropanoids (e.g. rosmarinic acid) and flavonoids (e.g. luteolin) from $P$. frutescens $(18,19)$, coumarins (e.g. imperatorin) from A. dahurica (20), coumarins (e.g. catechin) from A. catechu (21), triterpenoids (e.g. pachymic acid) from $P$. cocos (22), lignans (e.g. magnolol) from M. officinalis (23), sesquiterpenoids (e.g. atractylenolide I) from A. macrocephala (24), flavonoids (e.g. hesperidin) from C.reticulate (25), phenolic acid (e.g. homogentisic acid) and phenolic aldehyde (e.g. 3,4-dihydroxybenzaldehyde) from P.ternate (26), triterpenoids (e.g. platycodin D) from P. grandiflorum (27), triterpene saponin (e.g. glycyrrhizin) and flavonoids (e.g. liquiritin and liquiritigenin) from $G$. uralensis (28), flavonoids (e.g. spinosin and 6"'-feruloylspinosin) from Z. jujube (29), and phenols (e.g. 6-gingerol) from Z. officinale (30). Among those components, five compounds were investigated, including liquiritin and glycyrrhizin (G. uralensis), hesperidin (C. reticulata), rosmarinic acid (A. rugosa and $P$. frutescens), and 6-gingerol (Z. officinale) using HPLC-PDA. Consequently, hesperidin $(3.16 \mathrm{mg} / \mathrm{g})$ and glycyrrhizin $(3.03 \mathrm{mg} / \mathrm{g})$, marker compounds of $C$. reticulata and $G$. uralensis, respectively, were identified as the predominant components.

The inhibitory effects of GHJGS on inflammatory response were evaluated using the murine macrophage cell line, RAW 264.7. Macrophages are involved in the initiation, maintenance and resolution of inflammation $(31,32)$, and thus considered to be useful for inflammation-associated studies. Activated macrophages stimulate production of proinflammatory cytokines, such as TNF- $\alpha$ and IL-6 during pathological conditions of inflammatory disease (33). Significant increases in the production of TNF- $\alpha$ and IL-6 in LPS-stimulated RAW 264.7 cells were observed in the current study. GHJGS significantly inhibited TNF- $\alpha$ and IL- 6 production induced by LPS treatment. $\mathrm{PGE}_{2}$, a proinflammatory mediator, is produced through inflammatory stimulation of cyclooxygenase-2 (34). LPS stimulation significantly increased the level of $\mathrm{PGE}_{2}$, whereas GHJGS treatment markedly reduced LPS-mediated $\mathrm{PGE}_{2}$ production in RAW 264.7 cells. These results indicate the anti-inflammatory properties of GHJGS.

Production of proinflammatory factors, including TNF- $\alpha$, IL-6, and $\mathrm{PGE}_{2}$, is regulated by numerous intracellular signaling pathways at the transcription and post-transcription level (9). Inflammatory stimuli, such as LPS, activate MAPK and/or NF- $\mathrm{KB}$ signaling pathways associated with inflammatory cytokine production (35-37). In the present study, LPS stimulation markedly enhanced the levels of p-p38 MAPK, p-ERK and p-JNK, and nuclear expression levels of NF- $\mathrm{KB}$ p65 in RAW 264.7 cells. By contrast, GHJGS suppressed LPS-induced phosphorylation of p38 MAPK, ERK and JNK. However, NF- $\kappa$ B activation was not altered by GHJGS in LPS-treated RAW 264.7 cells.

Inflammation is associated with oxidative stress. During inflammation, ROS generation is a critical event in the elimination of pathogens (38), and induces production of proinflammatory cytokines and molecules $(32,39)$. Thus, the present study examined whether GHJGS has an inhibitory effect on ROS generation and determined that GHJGS suppressed LPS-induced ROS generation in macrophages. HO-1, a stress-inducible and redox-sensitive enzyme, is important during the inflammatory response (40). HO-1 negatively regulates production of proinflammatory cytokines, such as TNF- $\alpha$, IL-1 $\beta$ and IL- 6 in activated macrophages (41). Thus, HO-1 is a potential molecular target against inflammation and oxidative stress (42). The current study identified that GHJGS increased the expression of HO-1 in a dose-dependent manner.

In conclusion, the findings of the present study demonstrate that GHJGS inhibits LPS-stimulated production of proinflammatory biomarkers, TNF- $\alpha$, IL- 6 and $\mathrm{PGE}_{2}$ through suppression of the MAPK signaling pathway. Furthermore, GHJGS inhibited ROS generation and enhanced HO-1 expression. Overall, these findings confirm the anti-inflammatory and antioxidant actions of GHJGS, thus presenting it as a potential candidate for targeting inflammatory diseases and oxidative stress-associated diseases.

\section{Acknowledgements}

The present study was supported by the Korea Institute of Oriental Medicine (grant no. K15250).

\section{References}

1. Yun HS, Ryu BH, Park DW and Ryu KW: Experimental comparative studies on the effects of Kwakhyangjeonggisan and Souminkwakhyang-jeonggisan. K H Univ O Med J 21: 197-211, 1998 (In Korean).

2. Chuan-xing Y and Ling Z: Experimental researches on inhibitory effect of Huoxiang Zhengqi liquid on histamine release. Chin J Integr Med 9: 276-280, 2003 (In Chinese).

3. Xie C, Wang XF, Qi XJ, Lu LL and Chan HC: Effect of Huoxiang-zhengqi liquid on $\mathrm{HCO} 3$ - secretion by intact porcine distal airway epithelium. Sheng Li Xue Bao 60: 90-96, 2008 (In Chinese). 
4. Koo CM, Sun JK, Kim HH and Nam CG: Effects of GwakHyangJungGiSan on the arterial contraction in rabbit. Kor Orient Int Med 24: 260-268, 2003 (In Korean).

5. Zhang HK, Huang Y, Li K and Wu SH: Antibacterial material basis and quality control of Huoxiang Zhengqi tincture. Chin Tradit Herbal Drugs 43: 1349-1354, 2012 (In Chinese).

6. Ferrero-Miliani L, Nielsen OH, Andersen PS and Girardin SE: Chronic inflammation: Importance of NOD2 and NALP3 in interleukin-1beta generation. Clin Exp Immunol 147: 227-235, 2007.

7. Huerre MR and Gounon P: Inflammation: Patterns and new concepts. Res Immunol 47: 417-434, 1996.

8. Kalinski P: Regulation of immune responses by prostaglandin E2 J Immunol 188: 21-28, 2012

9. Saklatvala J, Dean J and Clark A: Control of the expression of inflammatory response genes. Biochem Soc Symp: 95-106, 2003.

10. Motterlini R, Foresti R, Bassi R and Green CJ: Curcumin, an antioxidant and anti-inflammatory agent, induces heme oxygenase-1 and protects endothelial cells against oxidative stress. Free Radic Biol Med 28: 1303-1312, 2000.

11. Liu H and Talalay P: Relevance of anti-inflammatory and antioxidant activities of exemestane and synergism with sulforaphane for disease prevention. Proc Natl Acad Sci USA 110: 19065-19070, 2013

12. Lee MY, Lee JA, Seo CS, Ha H, Lee H, Son J-K and Shin H-K: Anti-inflammatory activity of Angelica dahurica ethanolic extract on RAW264.7 cells via upregulation of heme oxygenase-1. Food Chem Toxic 49: 1047-1055, 2011.

13. Gurney SM, Scott KS, Kacinko SL, Presley BC and Logan BK Pharmacology, toxicology, and adverse effects of synthetic cannabinoid drugs. Forensic Sci Rev 26: 53-78, 2014

14. He M and Guo QL: Drug-induced fatal adverse effects in the United States from 1999 to 2004. Zhong Nan Da Xue Xue Bao Yi Xue Ban 33: 1060-1065, 2008 (In Chinese).

15. Szychowski J, Truchon JF and Bennani YL: Natural products in medicine: Transformational outcome of synthetic chemistry 57 9292-9308, 2014

16. Koeberle A and Werz O: Multi-target approach for natural products in inflammation 19: 1871-1882,2014.

17. Kim HK, Kim YA, Chun JM and Ko BS: Pattern analysis of Agastachis Herba and Pogostemonis Herba. Korean J Pharmacogn 34: 274-277, 2003 (In Korean).

18. Kim BY, Jeong JS, Kwon HJ, Lee JH and Hong SP: Determination of rosmarinic acid and caffeic acid from Perilla frutescens var. japonica and var. acuta by reversed-phase HPLC. Kor J Herbology 23: 67-72, 2008 (In Korean).

19. Jeon IH, Kim HS, Kang HJ, Lee HS, Jeong SI, Kim SJ and Jang SI: Anti-inflammatory and antipruritic effects of luteolin from Perilla (P. frutescens L.) leaves. Molecules 19: 6941-6951, 2014.

20. Li B, Zhang X, Wang J, Zhang L, Gao B, Shi S, Wang X, Li J and Tu P: Simultaneous characterisation of fifty coumarins from the roots of Angelica dahurica by off-line two-dimensional high-performance liquid chromatography coupled with electrospray ionisation tandem mass spectrometry. Phytochem Anal 25: 229-240, 2014

21. Wu Q, Yang Y and Simon JE: Qualitative and quantitative HPLC/MS determination of proanthocyanidins in areca nut (Areca catechu). Chem Biodivers 4: 2817-2826, 2007.

22. Li G, Xu ML, Lee CS, Woo MH, Chang HW and Son JK: Cytotoxicity and DNA topoisomerases inhibitory activity of constituents from the sclerotium of Poria cocos. Arch Pharm Res 27: 829-833, 2004

23. Jiang Y, Vaysse J, Gilard V, Balayssac S, Déjean S, Malet-Martino M, David B, Fiorini C and Barbin Y: Quality assessment of commercial Magnoliae officinalis Cortex by ${ }^{1} \mathrm{H}-\mathrm{NMR}$-based metabolomics and HPLC methods. Phytochem Anal 23: 387-395, 2012.

24. Tsai CJ, Liang JW and Lin HR: Sesquiterpenoids from Atractylodes macrocephala act as farnesoid $\mathrm{X}$ receptor and progesterone receptor modulators. Bioorg Med Chem Lett 22 2326-2329, 2012

25. Liu EH, Zhao P, Duan L, Zheng GD, Guo L, Yang H and Li P: Simultaneous determination of six bioactive flavonoids in Citri Reticulatae Pericarpium by rapid resolution liquid chromatography coupled with triple quadrupole electrospray tandem mass spectrometry. Food Chem 141: 3977-3983, 2013.
26. Han JH, Jo SG, Lee MJ, Baek SH and Park SH: Contents of homogentisic acid and 3,4-dihydroxybenzaldehyde in the Pinellia ternate by various processing method and its safety estimate. J Orient Physiol Pathol 18: 846-853, 2004 (In Korean).

27. Ha YW and Kim YS: Preparative isolation of six major saponins from Platycodi Radix by high-speed counter-current chromatography. Phytochem Anal 20: 207-213, 2009.

28. Zhang Q and Ye M: Chemical analysis of the Chinese herbal medicine Gan-Cao (licorice). J Chromatogr A 1216: 1954-1969, 2009

29. Niu C and Zhang J: Quantitative analysis and chromatographic fingerprinting of the semen zizyphi spinosae by ultra-high-performance liquid chromatography coupled with diode-array detector. J Sep Sci 34: 2989-2996, 2011.

30. Lee HR, Lee JH, Park CS, Ra KR, Ha JS, Cha MH, Kim SN, Choi Y, Hwang J and Nam JS: Physicochemical properties and antioxidant capacities of different parts of Ginger (Zingiber officinale Roscoe). J Korean Soc Food Sci Nutr 43: 1369-1379, 2014 (In Korean).

31. Ahmed JS and Mehlhorn H: Review: The cellular basis of the immunity to and immunopathogenesis of tropical theileriosis. Parasitol Res 85: 539-549, 1999.

32. Fujiwara $\mathrm{N}$ and Kobayashi $\mathrm{K}$ : Macrophages in inflammation. Curr Drug Targets Inflamm Allergy 4: 281-286, 2005.

33. Bottomley MJ, Webb NJ, Watson CJ, Holt PJ, Freemont AJ and Brenchley PE: Peripheral blood mononuclear cells from patients with rheumatoid arthritis spontaneously secrete vascular endothelial growth factor (VEGF): Specific up-regulation by tumour necrosis factor-alpha (TNF-alpha) in synovial fluid. Clin Exp Immunol 117: 171-176, 1999.

34. Crofford LJ, Wilder RL, Ristimäki AP, Sano H, Remmers EF, Epps HR and Hla T: Cyclooxygenase-1 and -2 expression in rheumatoid synovial tissues. Effects of interleukin-1 beta, phorbol ester, and corticosteroids. J Clin Invest 93: 1095-1101, 1994.

35. Kim KS, Cui X, Lee DS, Sohn JH, Yim JH, Kim YC and $\mathrm{Oh} \mathrm{H}$ : Anti-inflammatory effect of neoechinulin a from the marine fungus Eurotium sp. SF-5989 through the suppression of NF- $\kappa \mathrm{B}$ and p38 MAPK Pathways in lipopolysaccharide-stimulated RAW264.7 macrophages. Molecules 18: 13245-13259, 2013

36. Kwon DJ, Bae YS, Ju SM, Youn GS, Choi SY and Park J: Salicortin suppresses lipopolysaccharide-stimulated inflammatory responses via blockade of NF- $\mathrm{B}$ and JNK activation in RAW 264.7 macrophages. BMB Rep 47: 318-323, 2014.

37. Shao J, Li Y, Wang Z, Xiao M, Yin P, Lu Y, Qian X, $\mathrm{Xu} \mathrm{Y}$ and Liu J: 7b, a novel naphthalimide derivative, exhibited anti-inflammatory effects via targeted-inhibiting TAK 1 following down-regulation of ERK1/2- and p38 MAPK-mediated activation of NF- $\kappa \mathrm{B}$ in LPS-stimulated RAW264.7 macrophages. Int Immunopharmacol 17: 216-228, 2013.

38. Winyard PG, Ryan B, Eggleton P, Nissim A, Taylor E, Lo Faro ML, Burkholz T, Szabó-Taylor KE, Fox B, Viner N, et al: Measurement and meaning of markers of reactive species of oxygen, nitrogen and sulfur in healthy human subjects and patients with inflammatory joint disease. Biochem Soc Trans 39: 1226-1232, 2011

39. Singh KB, Maurya BK and Trigun SK: Activation of oxidative stress and inflammatory factors could account for histopathological progression of aflatoxin-B1 induced hepatocarcinogenesis in rat. Mol Cell Biochem 401: 185-196, 2015.

40. Ryter SW, Alam J and Choi AM: Heme oxygenase-1/carbon monoxide: From basic science to therapeutic applications. Physiol Rev 86: 583-650, 2006.

41. Southan GJ and Szabó C: Selective pharmacological inhibition of distinct nitric oxide synthase isoforms. Biochem Pharmacol 51: 383-394, 1996

42. Zabalgoitia M, Colston JT, Reddy SV, Holt JW, Regan RF, Stec DE, Rimoldi JM, Valente AJ and Chandrasekar B: Carbon monoxide donors or heme oxygenase-1 (HO-1) overexpression blocks interleukin-18-mediated NF-kappaB-PTEN-dependent human cardiac endothelial cell death. Free Radic Biol Med 44: 284-298, 2008 